\title{
Modélisation tri-dimensionnelle d'un disque céramique piézoélectrique chargé sur ses grandes faces
}

\section{BRISSAUD}

INSA, Laboratoire de Génie Electrique et Ferroélectricité, 69621 Villeurbanne cedex, France

This paper is devoted to the modelling of a piezoceramic disk loaded on its major surfaces by acoustic impedances denoted $\mathrm{Z}_{\mathrm{a}}$ and $\mathrm{Z}_{\mathrm{b}}$ respectively and using a three-dimensional approach. The stress, stain components and the displacements along the radius and the disk thickness are determined which allows the calculation of the electric impedance of the loaded disk and the related electromechanical coupling factors. It is shown that, due to lateral couplings, the resonance peak width of the 3D modelling is larger than that obtained for 1D models. Furthermore, due also to lateral couplings, there is no nodal plane inside the disk. It becomes a zero-phase plane that is, the displacements corresponding to this plane is minimal and the difference in phase related to the applied voltage is zero. This result is consistent with the previous finite element simulations.

\section{I - INTRODUCTION}

La détermination des fréquences de résonance d'un disque céramique piézoélectrique s'effectue généralement à partir de modèles uni-dimensionnels de type MASON [1] [2] ou K.L.M. [3] selon la nature de l'excitation : sinusoïdale pour le premier type et impulsionnelle pour le second. L'introduction des pertes électriques ou mécaniques dans ces modèles permet de prédire de manière correcte les diverses résonances des structures mono ou multi-couches [4]. Cependant, comme les couplages par effets Poisson et piézoélectriques sont négligés, les pertes calculées à partir de ces modèles peuvent être plus élevées que ne le laissent prévoir les caractéristiques des matériaux seuls et des colles [5]. Le but de ce travail est la prise en compte des couplages dans la modélisation analytique pour le calcul des modes de résonance d'un disque piézoélectrique chargé sur ces grandes faces par des impédances acoustiques quelconques $\mathrm{Z}_{\mathrm{a}}$ et $\mathrm{Z}_{\mathrm{b}}$. La démarche, similaire à celle développée pour le calcul des modes de résonance d'une plaque rectangulaire [5], repose sur l'utilisation de la modélisation tri-dimensionnelle utilisée pour la caractérisation des matériaux céramiques piézoélectriques [6].

Après la description du modèle utilisé nous présenterons les résultats issus des calculs théoriques que nous comparerons à ceux provenant de l'expérience.

\section{II - MODÉLISATION TRI.DIMENSIONNELLE}

Considérons un disque céramique piézoélectrique polarisé selon son épaisseur. Ses grandes faces sont chargées respectivement par les impédances acoustiques $\mathrm{Z}_{\mathrm{a}}$ et $\mathrm{Z}_{\mathrm{b}}$ et sa face latérale est libre. Les dimensions géométriques sont données à la figure 1 . Les axes de coordonnées étant des directions de propagation de mode pur, on peut considérer que les déplacements axiaux et radiaux sont de la forme :

$$
\mid \begin{aligned}
& u_{r}=U_{r} J_{1}(\alpha r) \exp j \omega t \\
& u_{z}=\left[A \sin \alpha_{3} z+B \cos \alpha_{3} z\right] \exp j \omega t
\end{aligned}
$$

où $\alpha=\omega / V$ et $V=C_{11}^{D} / \rho, \alpha_{3}=\omega / V_{3}$ et $V_{3}^{2}=C_{33}^{D} / \rho$ 
Compte tenu de (1) et (2) les composantes du tenseur des déformations se réduisent à $S_{\mathbf{r}}, S_{\theta}$, $S_{z}$ et $S_{4}=S_{5}=S_{6}=0$.

Par suite le tenseur des contraintes devient :

$$
\mid \begin{aligned}
& T_{r}=C_{11}^{D} S_{r}+C_{12}^{D} S_{\theta}+C_{13}^{D} S_{z}-h_{31} D_{3} \\
& T_{\theta}=C_{12}^{D} S_{r}+C_{11}^{D} S_{\theta}+C_{13}^{D} S_{z}-h_{31} D_{3} \\
& T_{z}=C_{13}^{D} S_{r}+C_{13}^{D} S_{\theta}+C_{33}^{D} S_{z}-h_{33} D_{3}
\end{aligned} \quad T_{4}=T_{5}=T_{6}=0
$$

De plus, il ne reste, pour les composantes du champ électrique, que la composante $\mathrm{E}_{3}$ soit :

$$
\mid \begin{aligned}
& E_{3}=-h_{31}\left(S_{r}+S_{\theta}\right)-h_{33} S_{z}+\beta_{33}^{S} D_{3} \\
& E_{1}=E_{2}=0 \text { et } D_{1}=D_{2}=0
\end{aligned}
$$

L'équation de Poisson appliquée à $D$ conduit à $D_{3}$ de la forme $D_{3}=D_{0} \exp j \omega t$ (6) où $D_{0}$ est une constante.

En écrivant maintenant la continuité des contraintes et des déplacements de part et d'autre des faces de la céramique on obtient le système linéaire $3 \times 3$ permettant le calcul des amplitudes $U_{r}, A$ et $B$ en fonction des constantes élastiques et piézoélectriques et aussi en fonction des impédances de charges $\mathrm{Z}_{\mathrm{a}}$ et $\mathrm{Z}_{\mathrm{b}}$ soit :

$$
\mid \begin{aligned}
& T_{1}(a)=0 \\
& T_{2}\left(a_{3}\right)=T_{a}=-Z_{a} \dot{u}_{a} \\
& \left.T_{Z_{2}}-a_{3}\right)=T_{b}=Z_{b} \dot{u}_{b}
\end{aligned} \quad \text { où } \dot{u}_{i}=\partial u_{i} / \partial t
$$

Les termes $\dot{\mathrm{u}}_{\mathrm{a}}$ et $\dot{\mathrm{u}}_{\mathrm{b}}$ sont les vitesses de déplacement dans les milieux A et $\mathrm{B}$. La résolution du système (6) conduit à :

$$
\mid \begin{aligned}
& \left.U_{r}=\left[h_{31} C_{33}^{D}(M P-N Q)+h_{33} C_{13}^{D}(M+N-P-Q)\right)\right] D_{0} / \alpha \Delta \\
& U_{r}=U_{r}^{\prime} D_{0} \\
& A=(P-N)\left[h_{33} J_{c}-h_{31} C_{13}^{D} J_{o}(\alpha a)\right] D_{o} /\left(\alpha_{3} \Delta \cos x_{3}\right)=A^{\prime} D_{0} / \alpha_{3} \cos x_{3} \\
& B=(M-Q)\left[h_{33} J_{c}-h_{31} C_{13}^{D} J_{o}(\alpha a)\right] D_{o} /\left(\alpha_{3} \Delta \sin x_{3}\right)
\end{aligned}
$$

avec

et

$$
\mid \begin{aligned}
& \Delta=J_{c} C_{33}^{D}(M P-N Q)+C_{13}^{D^{2}}(M+N-P-Q) \\
& J_{c}=C_{11}^{D} J_{o}(\alpha a)-\left(C_{11}^{D}-C_{12}^{D}\right) J_{1}(\alpha a) / \alpha a
\end{aligned}
$$

$$
\mid \begin{aligned}
& M=1+j \text { ra tg } x_{3} \\
& N=-1+j \text { ra cotg } x_{3} \\
& P=1-j \text { rb cotg } x_{3} \\
& Q=1+j \text { rb tg } x_{3}
\end{aligned}
$$

$$
\mid \begin{aligned}
& \mathrm{x}_{3}=\alpha_{3} \mathrm{a}_{3} \\
& \mathrm{ra}=\mathrm{Z}_{\mathrm{a}} / \mathrm{Z}_{\mathrm{c}} \\
& \mathrm{rb}=\mathrm{Z}_{\mathrm{b}} / \mathrm{Z}_{\mathrm{c}}
\end{aligned}
$$

En introduisant les relations (1) dans la relation (5) et en intégrant d'une part les charges sur la surface des électrodes puis le champ électrique suivant l'épaisseur du disque on obtient l'expression de l'impédance électrique du disque chargée. Elle s'écrit :

$$
Z=\frac{1}{j C_{0} \omega}\left[1-\frac{\alpha h_{31}}{\beta_{33}^{s}} U_{r}^{\prime} J_{o}(\alpha a)-\frac{h_{33} A^{\prime}}{\beta_{33}^{S}} \frac{\operatorname{tg} x_{3}}{x_{3}}\right]
$$


où $\mathrm{C}_{0}=\varepsilon_{33}^{\mathrm{S}} \pi \mathrm{a}^{2} / 2$ a3 est la capacité du disque chargée.

En reportant dans la relation (12) les expressions (7) et (8) de $U_{r}^{\prime}$ et $A^{\prime}$, on peut mettre l'impédance du disque sous la forme:

$$
\mathrm{Z}=\mathrm{R}+\mathrm{jX}
$$

On constate alors que les parties réelles et imaginaires sont fonction de $\mathrm{Z}_{\mathrm{a}}$ et $\mathrm{Z}_{\mathrm{b}}$.

\section{III - DISCUSSION}

La relation (9) de l'impédance du disque chargée intègre sous un même formalisme les modes de résonance radiale et axiale de l'ensemble. Les résonances radiales apparaissent lorsque $\Delta$ devient nul ou minimal selon que les pertes sont ou ne sont pas prises en compte.

Si le matériau est sans pertes on retrouve l'expression obtenue pour un disque libre [6]. Cette dernière se simplifie encore si on suppose que les couplages élastiques $\left(\mathrm{C}_{13}\right)$ et piézoélectriques (h33) sont nuls. On obtient dans ce cas une relation similaire à celle du disque libre vibrant en radial [7].

Les résonances axiales se produisent lorsque tg $x_{3}$ devient $\infty$ comme pour le disque non chargé.

À partir des relations donnant $\mathrm{U}_{\mathrm{r}}$ et $\mathrm{A}^{\prime}$ il est possible de faire apparaître les coefficients de couplage $\mathrm{k}_{\mathrm{L}}$ et $\mathrm{k}_{\mathrm{T}}$ qui s'expriment par :

$$
\mathrm{k}_{\mathrm{L}}^{2}=\mathrm{h}_{31}^{2} / \beta_{33}^{\mathrm{S}} C_{11}^{\mathrm{D}} \text { et } \mathrm{k}_{\mathrm{T}}^{2}=\mathrm{h}_{33}^{2} / \beta_{33}^{\mathrm{S}} \mathrm{C}_{33}^{\mathrm{D}}
$$

Ces coefficients de couplages sont les coefficients de couplages intrinsèques. Ce ne sont pas ceux que l'on peut obtenir par une mesure directe de fréquence [7] car ils n'interviennent pas seuls dans l'expression de l'impédance.

Le calcul théorique de l'évolution du déplacement uz selon l'épaisseur du disque piézoélectrique montre qu'il n'existe pas de plan nodal. Par contre il existe un plan pour lequel le déplacement uz est minimal et en phase avec le courant d'excitation $i$. L'abscisse $\mathrm{x}_{0}$ de ce plan est donnée par la relation

$$
\operatorname{tg} \alpha_{3} x_{o}=\frac{Z_{b}-Z_{a}}{Z_{b}+Z_{a}} \operatorname{tg} \alpha_{3} a_{3}
$$

c'est exactement la même que celle obtenue pour une plaque rectangulaire [5]. Ces résultats sont en accord avec les mesures expérimentales et les simulations par éléments finis de ces structures [8].

\section{IV - RÉSULTATS EXPÉRIMENTAUX}

La figure 2 donne, pour un disque céramique de $50 \mathrm{~mm}$ de diamètre et $5 \mathrm{~mm}$ d'épaisseur et de type PZT classique, l'évolution expérimentale de son impédance électrique lorsqu'il est libre. Les résonances radiales et axiales se produisent respectivement à $88,5 \mathrm{kHz}$ et $417,3 \mathrm{kHz}$. La figure 3 montre pour le même disque l'évolution de son impédance électrique expérimentale lorsqu'il est chargé sur une face par de l'eau et de l'air sur l'autre face. La résonance radiale n'a pas été modifiée, par contre l'amplitude du mode axiale a été réduite de manière importante. Les résultats de simulations correspondant sont représentés figure 4 et 5 . Les pertes dans le matériau piézoélectrique ont été introduites dans la simulation. Compte tenu des approximations faites dans l'établissement du modèle théorique, la concordance entre les résultats théoriques et expérimentaux est satisfaisante.

\section{V - CONCLUSION}

Nous avons montré que le calcul de l'impédance électrique d'un disque piézoélectrique chargé est possible en utilisant une approche tri-dimensionnelle prenant en compte les couplages élastiques et piézoélectriques et les pertes dans le matériau. Cette modélisation plus réaliste que celle de MASON, met bien en évidence l'influence des modes radiaux et des impédances de charge sur les modes axiaux.

\section{BIBLIOGRAPHIE}

[1] MASON W.P. - Proc. IRE, vol. 23 - Oct. 1935.

[2] IEEE Standards on Piezoelectricity. Special issue, vol. SU 31, n 2, II, 1984.

[3] KRIMHOLTZ R., LEEDOM D. A., MATTHAEI G. L. - Electronics Letters, vol. 6, ${ }^{\circ}$ 13, June 1971.

[4] DION J. L., BRISSAUD M. - 2ème Congrès Français d'Acoustique. Supp. J. Physique, vol. 2 C1.373-376, Avril 1992. 
[5] BRISSAUD M. - Ultrasonics Inter. Wien Juillet 1993.

[6] BRISSAUD M. - IEEE, Trans. UFFC, vol. 38, $\mathrm{n}^{\circ} 6,1991,603-617$.

[7] BERLINCOURT et al. - In Physical Acoustics, Academic Press, vol. 1, part. A, 1964.

[8] DECARPIGNY J. N. - Thèse Doc ès Sciences. Université Sciences et Techniques de Lille (1984).

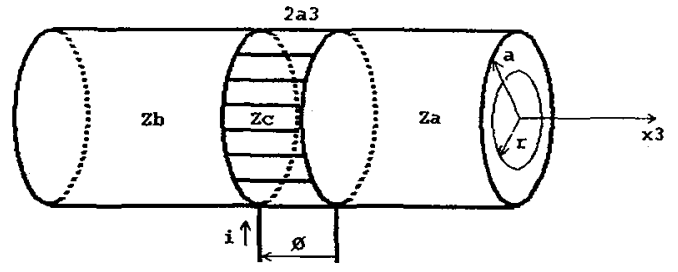

Fig. 1 Disque céramique chargé : notations et dimensions

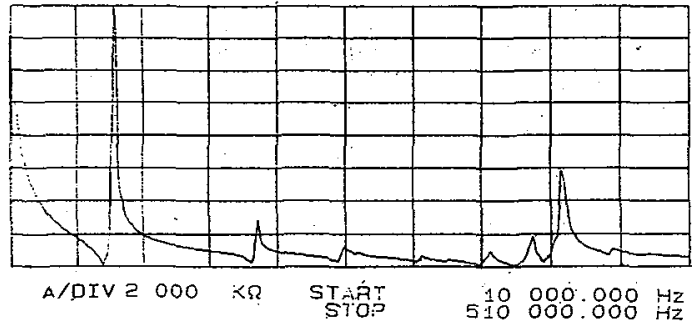

Fig. 2 Évolution expérimentale de l'impédance du disque libre en fonction de la fréquence

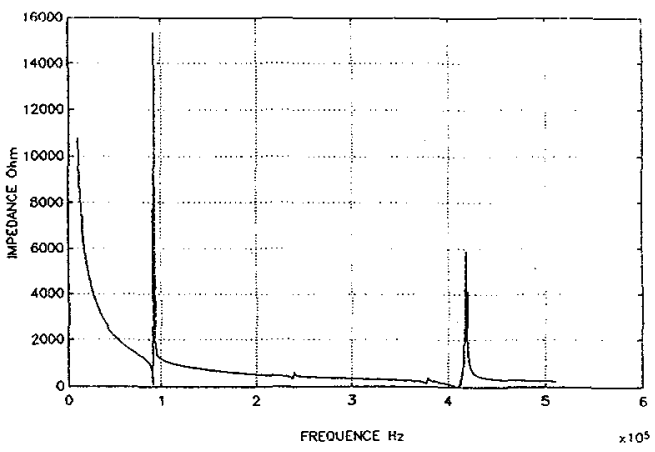

Fig. 4 Courbe simulée de l'impédance du disque libre (figure I)

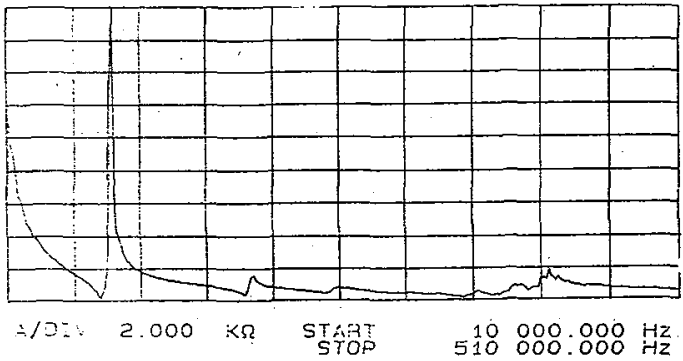

Fig. 3 Évolution expérimentale de l'impédance du disque. chargé par de l'eau et de l'air

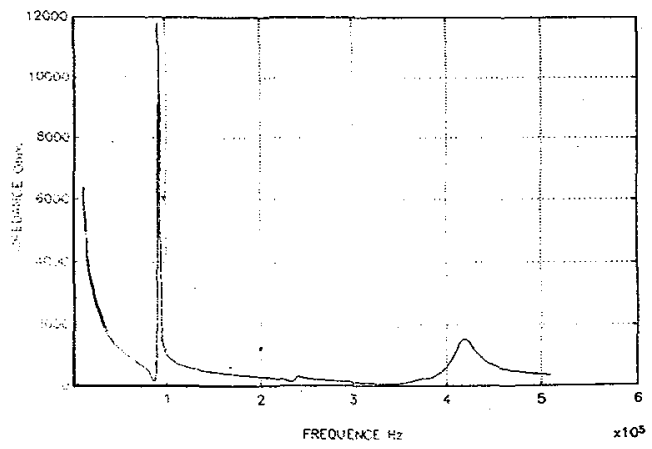

Fig. 5 Courbe simulée de l'impédance du disque chargé par de l'eau et de l'air 